\title{
Utilización de Classroom en la Universidad Tecnológica de Tehuacán (UTTeh)
}

\section{Classroom use at the Universidad Tecnológica de Tehuacán (UTThe)}

\author{
ALFARO-HERRERA, Julio César†, VÁZQUEZ-LÓPEZ, José Antonio, GALICIA-GARCÍA, Christian \\ y SÁNCHEZ-DELGADO, Octavio
}

Universidad Tecnológica de Tehuacán, Prolongación de la 1 sur No. 1101 San Pablo Tepetzingo, 75859 Tehuacán, Pue.

ID $1^{\text {er }}$ Autor: Julio César, Alfaro-Herrera / ORC ID: 0000-0003-2500-6698, CVU CONACYT ID: 515414

ID 1 ${ }^{\text {er }}$ Coautor: José Antonio, Vázquez-López / ORC ID: 0000-0002-4497-8030, CVU CONACYT ID: 1014004

ID $3^{\text {er }}$ Coautor: Christian, Galicia-García / ORC ID: 0000-0001-7796-4295, CVU CONACYT ID: 954206

ID $3^{\text {er }}$ Coautor: Octavio, Sánchez Delgado / ORC ID: 0000-0002-7073-0522, CVU CONACYT ID: 957303

DOI: $10.35429 / J I T C .2019 .7 .3 .19 .29$

Recibido 06 de Enero, 2019; Aceptado 30 de Marzo, 2019

\section{Resumen}

Esta investigación, tiene como objetivo identificar el impacto de las Tecnologías de Información mediante la utilización de la plataforma Google Classroom en el proceso de enseñanza aprendizaje, además del fortalecimiento y desarrollo de las competencias digitales en los estudiantes de los diferentes Programas Educativos (PE), tanto a nivel Técnico Superior Universitario (TSU) como a nivel Ingeniería de la Universidad Tecnológica de Tehuacán (UTT); de igual manera, se busca identificar cómo la plataforma Classroom apoya el quehacer docente desde el punto de vista de agilizar la entrega/recepción/calificación de diversas actividades generadas por el profesor con cada uno de sus grupos. Para tal fin se aplicaron dos instrumentos de medición de manera digital (encuesta en formularios de Google Forms), uno se aplica a los alumnos, tomando una muestra representativa de los grupos de cada PE (siete) en ambos niveles educativos (TSU e Ingeniería); otro instrumento se aplica a una muestra representativa de docentes de la institución. Los resultados obtenidos indican que el 92.9\% de los estudiantes y el $80.8 \%$ de los profesores encuestados han utilizado la plataforma Google Classroom, revelando una gama amplia de aplicaciones y opiniones con respecto a la utilización y beneficios de Classroom en la UTT.

Tecnologías de Información, Google Classroom, Competencias digitales, Docente, Alumno

\begin{abstract}
This research aims to identify the impact of Information Technology through the use of Google Classroom Platform in the teaching-learning process, it also aims to strengthen and develop digital skills in students of the different Educational Programs (EP), both at the undergraduate level and at engineering level of the Technological University of Tehuacán (UTT), in addition, it is sought to identify how Classroom Platform supports teaching tasks such as: speeding the delivery, reception and grading of various activities generated by the teacher with every group. For this purpose, two measuring instruments were digitally applied (Google Forms survey). This one is applied to students, taking a representative sample of the groups of each EP (seven) in both educational levels (undergraduate and engineering level). Another instrument was applied to a representative sample of teachers of the school. The results show that the $92.9 \%$ of the students and the $80 \%$ of the teachers that were interviewed have used Google Classroom, revealing a wide range of applications and opinions regarding the use and benefits of Google Classroom at UTT
\end{abstract}

Information Technology, Google Classroom, Digital skills, Teacher, Student

Citación: ALFARO-HERRERA, Julio César, VÁZQUEZ-LÓPEZ, José Antonio, GALICIA-GARCÍA, Christian y SÁNCHEZ-DELGADO, Octavio. Utilización de Classroom en la Universidad Tecnológica de Tehuacán (UTTeh). Revista de Tecnologías de la Información y Comunicaciones. 2019. 3-7: 19-29

\footnotetext{
$\dagger$ Investigador contribuyendo como primer autor.
} 


\section{Introducción}

La UTT es una universidad pública, se ubica en San Pablo Tepetzingo, población que pertenece al municipio de Tehuacán, Puebla; actualmente cuenta con nueve PE en el nivel de Técnico superior universitario: Agricultura Sustentable y Protegida, Desarrollo de Negocios Área Mercadotecnia, Energías Renovables Área Calidad y Ahorro de Energía, Mecatrónica Área Sistemas de Manufactura Flexible, Procesos Alimentarios, Procesos Industriales Área Automotriz, Tecnologías de la Información (TI) Área Desarrollo de Software Multiplataforma y Área Infraestructura de Redes Digitales y Mantenimiento Industrial; seis ingenierías Agricultura Sustentable y Protegida, Energías Renovables, Mecatrónica, Procesos Bioalimentarios, Procesos y Operaciones Industriales y Tecnologías de la Información y dos licenciaturas Enfermería e Innovación de Negocios y Mercadotecnia.

La UTT aplica el modelo de competencias profesionales, entendiendo que "la competencia profesional es el conjunto de las habilidades, destrezas, actitudes y conocimientos teórico prácticos que requiere un profesional de cualquier disciplina" (CENEVAL, 2000). Por lo tanto, siendo una universidad de vanguardia, genera las condiciones para que todos sus estudiantes destaquen en el aspecto personal y profesional, apoyando la productividad académica mediante la entrega recepción de información digital y a la par cuidando el medio ambiente.

Para tal propósito, es fundamental la participación del personal docente, y en ese sentido la UTT ha llevado a cabo una serie de capacitaciones dirigidas a un porcentaje considerable de maestros adscritos a los diferentes PE en relación al uso de herramientas tecnológicas aplicadas a la educación. De tal manera que el presente artículo aborda el uso de Classroom como herramienta tecnológica.

\section{Justificación}

El modelo educativo de la Universidades Tecnológicas y por ende de la UTT, específica que las asignaturas que se imparten deben ser el $70 \%$ prácticas y $30 \%$ teóricas a nivel TSU, y en el nivel ingeniería $60 \%$ prácticas y $40 \%$ teóricas.
Por lo tanto, cualquier PE que se oferte en la institución no puede ser impartido de forma virtual, pero si tiene la opción de emplear recursos como el Blended Learning, que consiste "en una combinación de la enseñanza presencial y de la enseñanza virtual. Es una muy buena opción para los profesores para traer elementos al campo presencial en vez de simplemente usar materiales digitales como elementos complementarios del curso (que se usan casi siempre en casa)" (Collazos, 2019). El diagrama siguiente representa de forma clara los elementos que conforman la implementación del Blended Learning (Figura 1).
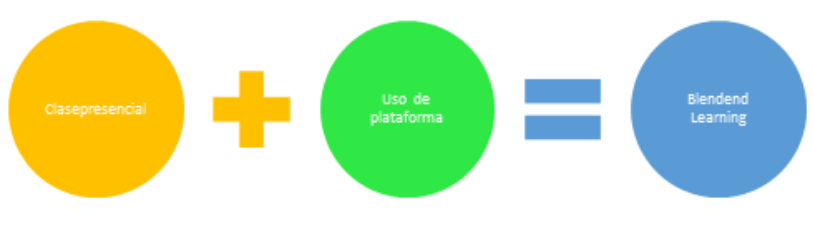

Figura 5 Blended Learning

Este esquema de trabajo permite a los profesores brindar diversos materiales a los estudiantes, así como asignar y calificar tareas, aplicar exámenes en línea, intercambiar ideas a través de foros, colocar anuncios y sugerencias, fomentando -tanto en los estudiantes como en el profesores- el desarrollo y fortalecimiento de las competencias digitales y una cultura ecológica sustentable, ya que permite un ahorro significativo en papel y tinta.

\section{Planteamiento del problema}

La UTT se encuentra inscrita como un centro educativo ante Google Suite, uno de los múltiples beneficios que brinda esta plataforma, es que permite a los miembros de la institución, crear, administrar y cerrar clases en la plataforma Classroom, sin embargo todo parece indicar que este beneficio no ha sido aprovechado al $100 \%$ por los profesores, pues a decir de algunos, solo utilizan Classroom como un repositorio de trabajos, es decir, para la función básica de asignar y recibir tareas, además, un número significativo de ellos, argumenta desconocer o no contar con la suficiente preparación para explotar todas las opciones (herramientas) que brinda la plataforma en comento. 
Por otro lado, no se tiene la certeza de si el estudiante conoce y maneja esta plataforma, en qué medida interactúa con los miembros de la clase través de ella, si está familiarizado con las diferentes opciones que la integran, la facilidad o dificultad para utilizarla, el beneficio o perjuicio que representa su implementación en la asignatura y si requiere alguna capacitación adicional para su correcta utilización.

Al determinar esta falta de información o problema se decidió llevar a cabo una investigación de campo mediante la aplicación de un instrumento electrónico de recolección de datos (aplicar una encuesta mediante un formulario de Google); con la finalidad de dar certeza a la presente investigación se recurrió a la aplicación de la estadística tomando una muestra representativa de la población aplicando la fórmula para calcular el tamaño de la muestra debido a que se conoce el tamaño de la población.

\section{Objetivos}

\section{Objetivo General}

Determinar la utilidad de las Tecnologías de Información mediante el empleo de la plataforma Google Classroom en el proceso de enseñanza aprendizaje tanto para profesores como estudiantes de los diferentes programas educativos en la UTT.

\section{Objetivos específicos}

Estructurar una encuesta que permita la obtención de datos fundamentales, basada en la objetividad, concisión y claridad, susceptible de ser aplicada tanto a profesores como estudiantes de la UTT.

Recopilar datos fehacientes a través de la aplicación digital de la técnica encuesta, previa determinación de la población muestra en los programas educativos que conforman la UTT.

Identificar las áreas de oportunidad desde el punto de vista tecnológico, tanto de estudiantes como de profesores, en lo que se refiere al uso de Classroom como herramienta práctica en el proceso de enseñanza aprendizaje.

\section{Marco teórico}

\section{Tecnologías de Información}

"La información puede estar en papel, en medios digitales, grabada o en cualquier otro dispositivo $\mathrm{u}$ objeto que permita guardarla o transmitirla. Las tecnologías de información son aquellas herramientas o medios que ayudan a las personas a tener una interacción utilizando medios basados en tecnología, para lograr esta interacción se necesita un emisor, un receptor de información y un canal que se le llama "comunicaciones" por lo que algunos autores se refieren a ellas como:" (AYALA, 2013)

"La TI proporciona la base, o plataforma, sobre la cual la empresa puede construir sus sistemas de información específicos" (LAUDON Kenneth C., 2008).

\section{Blended Learning (B Learning)}

La definición que resume en unas cuantas palabras lo que significa B Learning es la siguiente: "which combines face-to-face and virtual teaching (combina la enseñanza virtual con la presencial)" (Coaten, 2019)

\section{Dirección de proyectos}

“dirección de proyectos es la aplicación de conocimientos, habilidades, herramientas y técnicas a las actividades del proyecto para cumplir con los requisitos del mismo. Se logra mediante la aplicación e integración adecuadas de los 47 procesos de la dirección de proyectos, agrupados de manera lógica, categorizados en cinco Grupos de Procesos. Estos cinco Grupos de Procesos son:" (Institute, 2013)

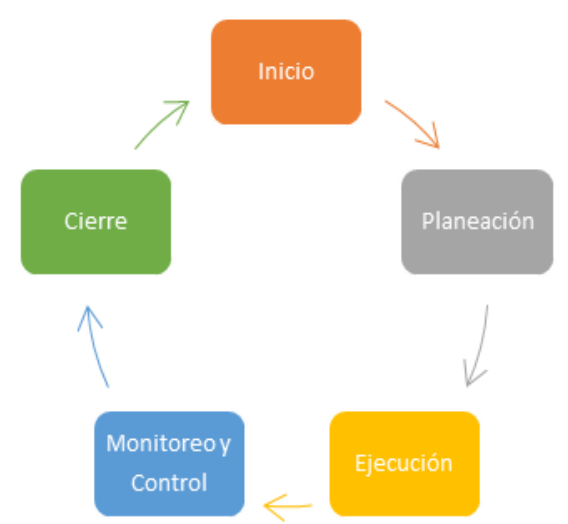

Figura 6 Grupos de procesos del proyecto 


\section{Google Classroom}

"Es un servicio gratuito para escuelas, ONG y cualquier persona que tenga una cuenta de Google personal. Classroom facilita la conexión entre profesores y alumnos, tanto dentro como fuera de la escuela. Classroom permite ahorrar tiempo y papel, así como crear clases, distribuir tareas, comunicarse con otros usuarios y mantener el trabajo organizado con facilidad" (Google, 2019).

\section{Muestreo probabilístico}

Consiste en que "todos los individuos tienen la misma probabilidad de ser elegidos para formar parte de una muestra $y$, consiguientemente, todas las posibles muestras de tamaño $\mathrm{n}$ tienen la misma probabilidad de ser seleccionadas. Sólo esos métodos de muestreo probabilísticos nos aseguran la representatividad de la muestra extraída y son, por tanto, los más recomendables." (Matemáticas, 2019).

\section{Instrumento de recolección de datos}

El instrumento más utilizado para la recolección de datos es la encuesta, que debe tener contener preguntas cerradas que cumplan por lo menos las siguientes características: ser claras, precisas y comprensibles; breves; formuladas en lenguaje simple, directo y familiar. (Sampieri, 2014)

\section{Metodología a utilizar}

La presente investigación se llevó a cabo entre académicos del PE de TIC y del PE de Procesos Industriales, por lo tanto, para su desarrollo y aplicación se utilizó una metodología basada en proyectos que indica el PMBOK, aplicando cada una de las fases conforme a lo siguiente:

1. Inicio: Esta primera etapa se describió el alcance del proyecto, las metas generales y se establecieron los participantes (equipo involucrado). Además de aterrizar la complejidad, características, tiempo dedicado para el desarrollo del proyecto.

2. Planeación: en esta segunda etapa se establecieron detalladamente las tareas y actividades a desarrollar por parte del equipo de trabajo. En la cual se diseñó el instrumento de evaluación, estableciendo la logística para su correcta aplicación.
3. Ejecución: consiste en aplicar el instrumento a una muestra proporcional de cada PE, conforme a la aplicación de la fórmula del muestreo probabilístico.

4. Monitoreo y control: se verificó que se aplicara el instrumento conforme a lo planeado.

5. Cierre: generar los resultados y conclusiones de la presente investigación.

\section{Desarrollo}

\section{Realización de la encuesta para los estudiantes}

La encuesta se desarrolló en plataforma Google Forms conforme a las siguientes secciones:

4.1.1. Datos generales del estudiante: en la cual se solicita la edad y el nivel de estudios actual, esto con la finalidad de establecer parámetros que influyen o no en el conocimiento y empleo de los medios digitales, en específico de la plataforma Classroom.

\section{Utilización de Classroom en la Universidad Tecnológica de Tehuacán (UTTeh)}

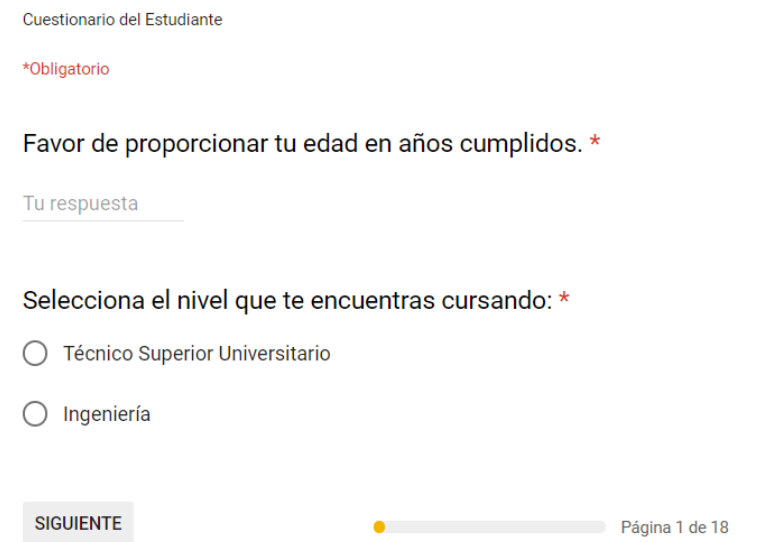

Figura 7 Datos generales del estudiante

4.1.2. PE: mediante una lista desplegable elige el programa de estudios al que pertenece el estudiante, un dato que permite establecer el nivel de aplicación de la plataforma en las diferentes carreras que conforman la UTT.

Aquí se separa en nivel TSU, desplegando los PE pertenecientes a este apartado. 


\section{Técnico Superior Universitario}

Favor de elegir el programa educativo al que perteneces: * Elige

ATRÁs

SIGUIENTE

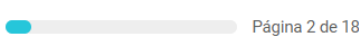

Figura 8 PE de pertenencia en TSU

En el nivel ingeniería, se despliegan las respectivas carreras indicadas en el apartado 1. Introducción.

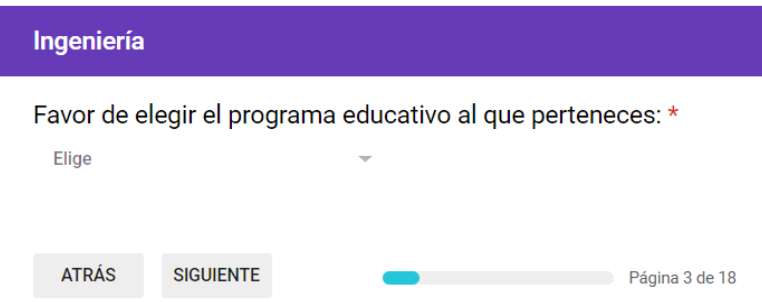

Figura 9 PE de pertenencia en Ing.

4.1.3 Se pregunta si el estudiante utiliza la plataforma Classroom.

1. ¿Utilizas la plataforma Classroom?

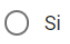

No

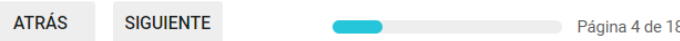

Figura 10 Utiliza Classroom

En caso de responder $\mathrm{Si}$, se continua con el punto 4.1.4, en caso de que la respuesta es no, el presente estudio se limita a preguntar si ha ocupado otro tipo de plataforma educativa.

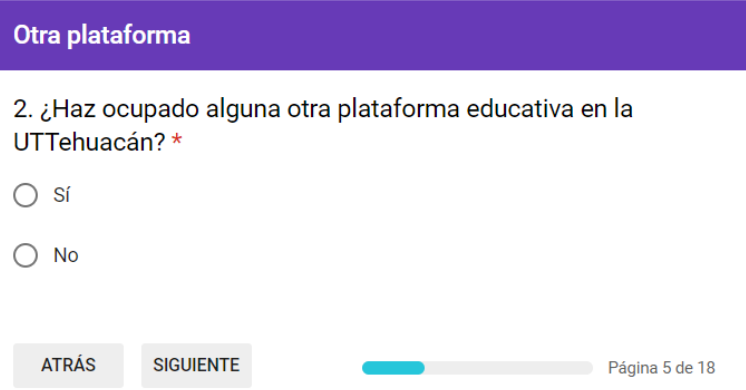

Figura 11 Utilización de otra plataforma
En caso de responder que, si ha utilizado otra plataforma educativa, se le pregunta que especifique cuál plataforma ha utilizado, proporcionada la información se proceda a agradecer su participación y se finaliza la aplicación de la encuesta.

\section{Otra plataforma}

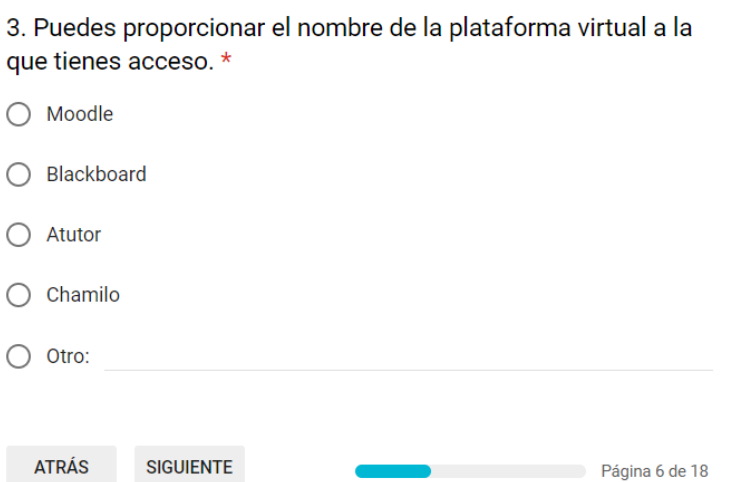

Figura 12 Nombre de la otra plataforma que utiliza

4.1.4 $\mathrm{Al}$ responder de forma positiva a la pregunta 1 , se procede a preguntar qué le parece la usabilidad de la plataforma Classroom.

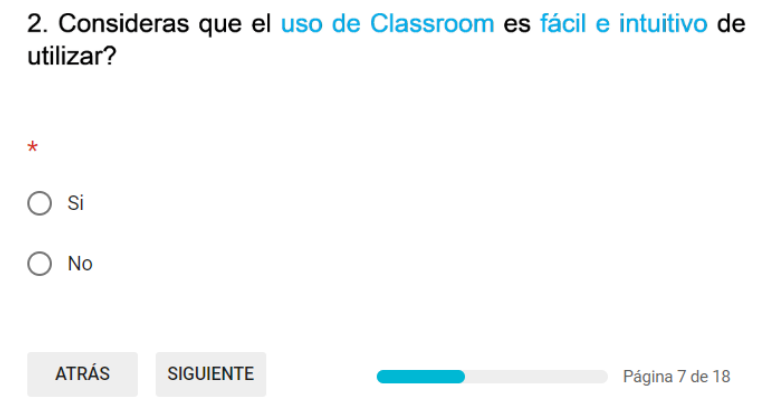

Figura 13 Usabilidad de Classroom

En caso afirmativo se pasa a la pregunta del apartado 4.1.5, en caso contrario se indaga sobre que aspecto en particular se le dificulta de la plataforma Classroom.

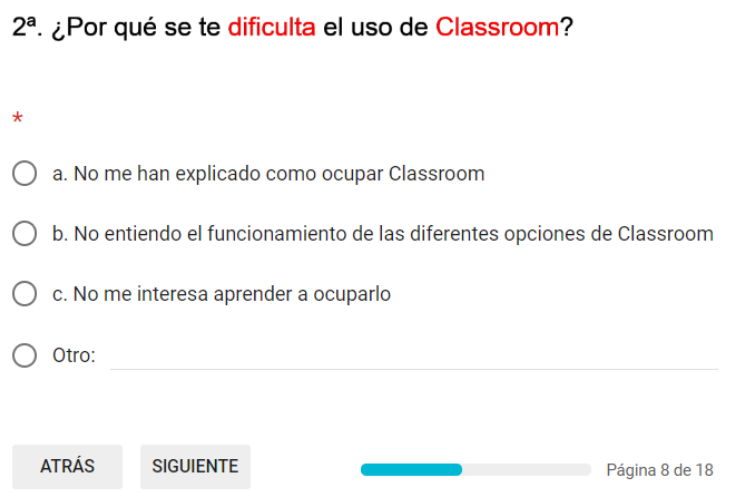

Figura 14 Dificultad de Classroom

ALFARO-HERRERA, Julio César, VÁZQUEZ-LÓPEZ, José Antonio, GALICIA-GARCÍA, Christian y SÁNCHEZ-DELGADO, Octavio. Utilización de Classroom en la Universidad Tecnológica de Tehuacán (UTTeh). Revista de Tecnologías de la Información y Comunicaciones. 2019 
4.1.5 La siguiente pregunta va relacionada con la frecuencia que los estudiantes utilizan Classroom.

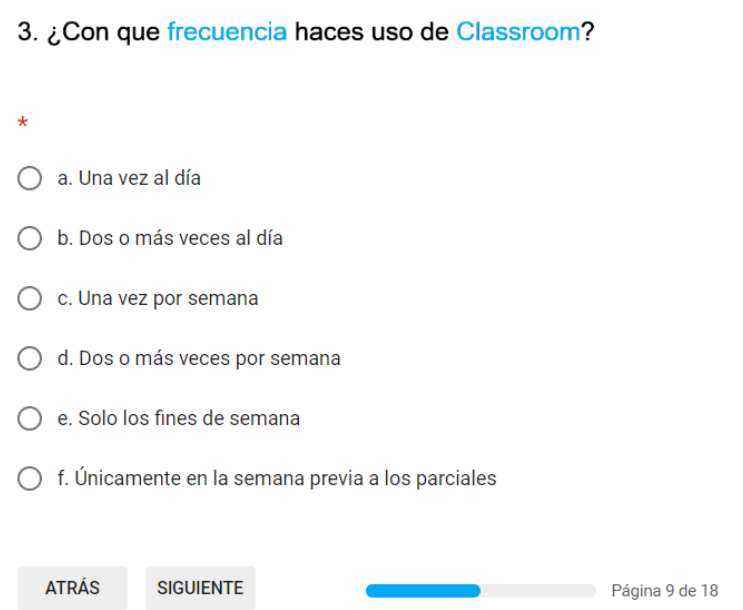

Figura 15 Frecuencia al utilizar Classroom

4.1.6 Posteriormente se preguntó por qué considera útil el uso de Classroom, proporcionando las opciones básicas de funcionamiento de la plataforma.

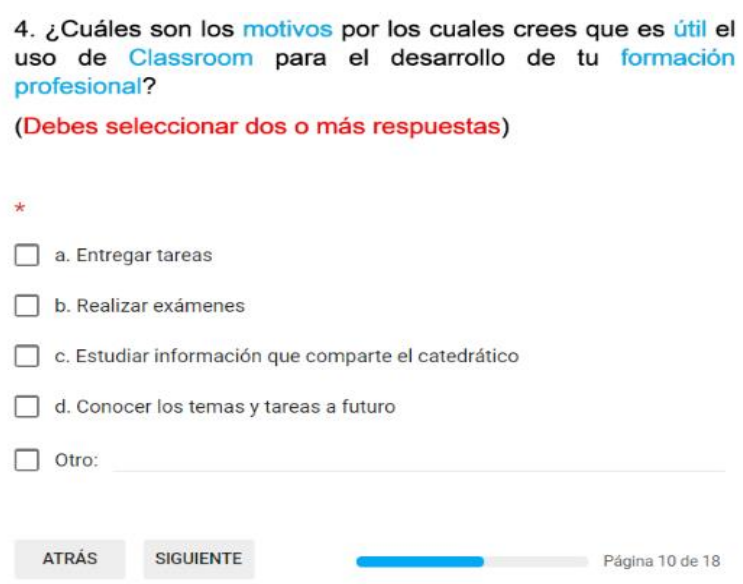

Figura 16 Utilidad de Classroom

4.1.7 La siguiente pregunta va relacionada con los beneficios que representa el uso de Classroom como herramienta tecnológica.

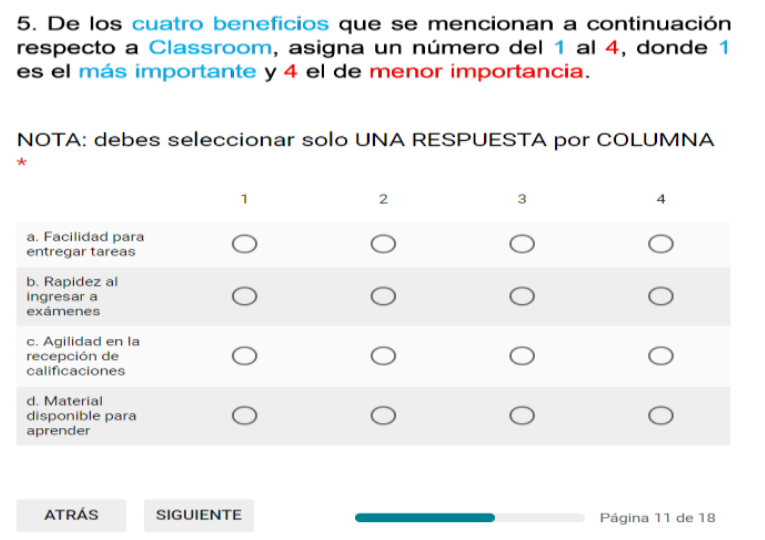

Figura 17 Beneficios de Classroom

ISSN 2531-2200

ECORFAN ${ }^{\circledR}$ Todos los derechos reservados
4.1.8 El siguiente reactivo habla sobre el uso de los foros en Classroom, opción que facilita el intercambio de ideas entre los participantes.

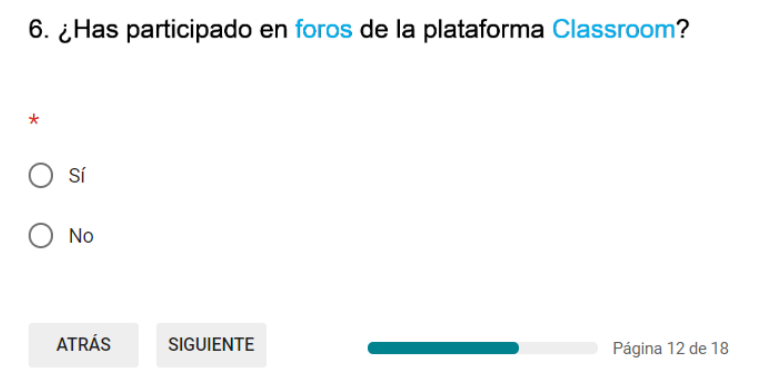

Figura 18 Foros de Classroom

4.1.9 El cuestionamiento siguiente va la relacionado con el acceso a Classroom.

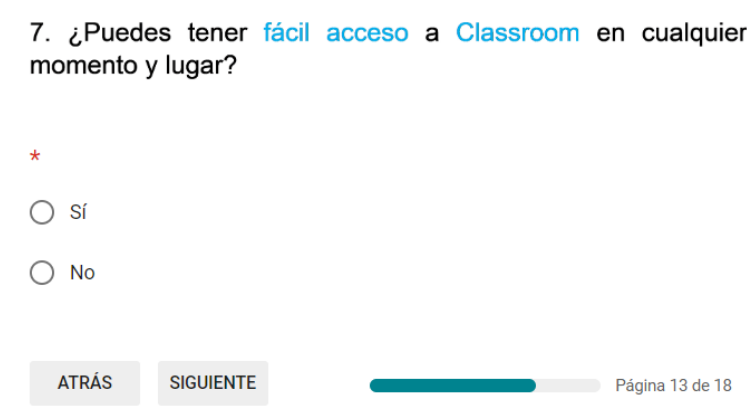

Figura 19 Acceso a Classroom

En caso de que la respuesta sea afirmativa se dirige al apartado 4.1.10, pero en caso que la respuesta sea negativa se indaga para saber que obstaculiza el acceso a Classroom.

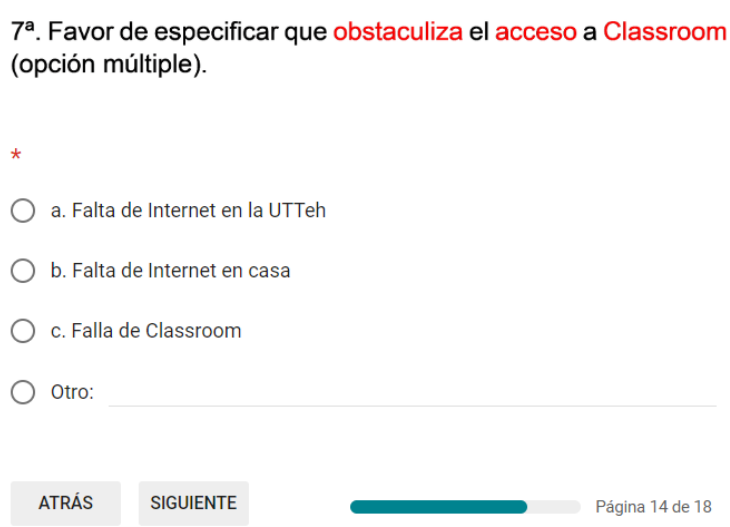

Figura 20 Obstáculo de acceso a Classroom

4.1.10 Posteriormente se busca identificar que dificultad se presenta al momento de entregar o enterar alguna tarea o actividad. 
8. ¿Se te ha presentado alguna dificultad o percance a la hora de presentar un trabajo o un examen en Classroom?
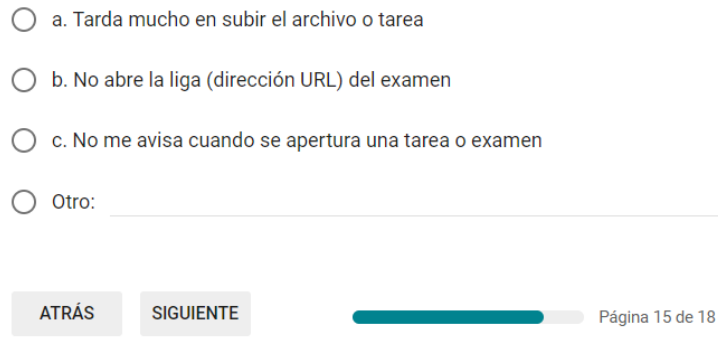

Figura 21 Dificultad entrega de actividad

4.1.11 Este cuestionamiento pretende identificar qué tanto contribuye el emplear Classroom en las diversas actividades académicas o de información o evaluación en el ámbito escolar.

9. ¿El empleo de Classroom te facilita la realización de actividades escolares, como es la entrega de tareas y aplicación de exámenes?

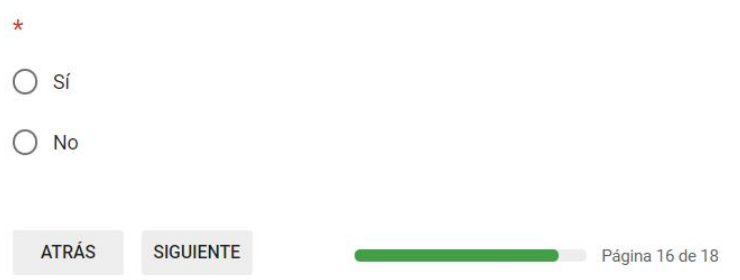

Figura 22 Empleo de Classroom

4.1.12 Por último se pregunta el nivel de satisfacción que se tiene al utilizar la plataforma Classroom, empleando una escala numérica y en su casos aplicar la escala de Likert.

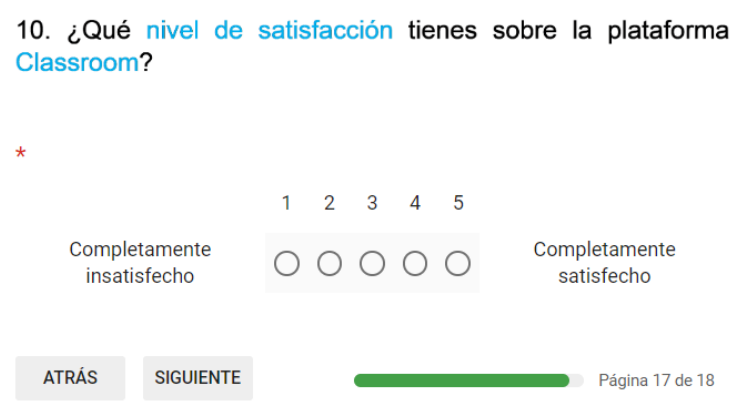

Figura 23 Nivel de satisfacción

4.1.13 Se concluye la encuesta con el agradecimiento por la participación en la encuesta.

\section{Utilización de Classroom en la Universidad Tecnológica de Tehuacán (UTTeh)}

¡Gracias por tu participación!

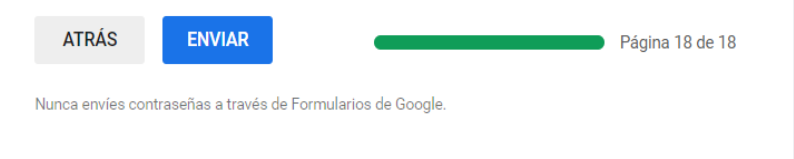

Figura 24 Agradecimiento por participación

\section{Realización de la encuesta para los docentes}

La encuesta aplicada a los profesores se realiza de la misma forma que a los estudiantes, se desarrolla en plataforma Google Forms conforme a las siguientes secciones:

4.2.1 Se solicitan los datos generales del docente como rango de edad, PE y nivel académico donde imparte la mayor cantidad de horas.

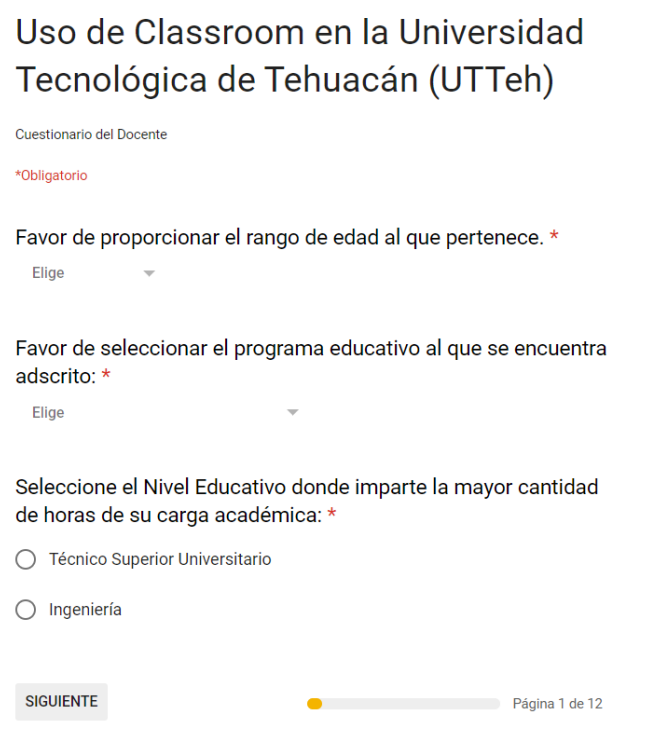

Figura 25 Datos generales del docente

4.2.2 Posteriormente se cuestiona si emplea la plataforma Classroom.

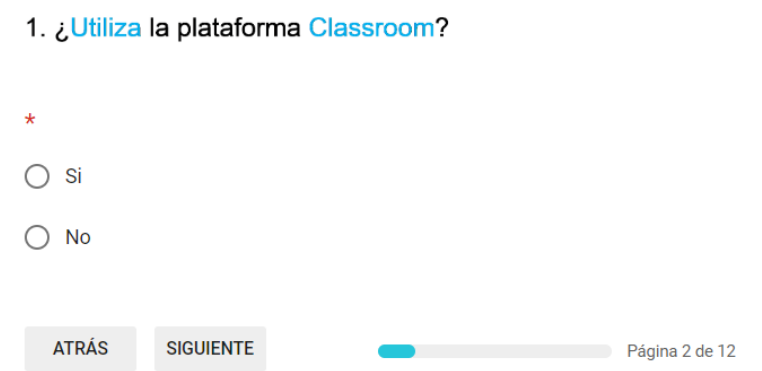

Figura 26 Utilización de Classroom

ALFARO-HERRERA, Julio César, VÁZQUEZ-LÓPEZ, José Antonio, GALICIA-GARCÍA, Christian y SÁNCHEZ-DELGADO, Octavio. Utilización de Classroom en la Universidad Tecnológica de Tehuacán (UTTeh). Revista de Tecnologías de la Información y Comunicaciones. 2019 
En caso de que la respuesta sea positiva se va al apartado 4.2.3, pero en caso negativo se procede a preguntar si ocupa algún otro tipo de plataforma.

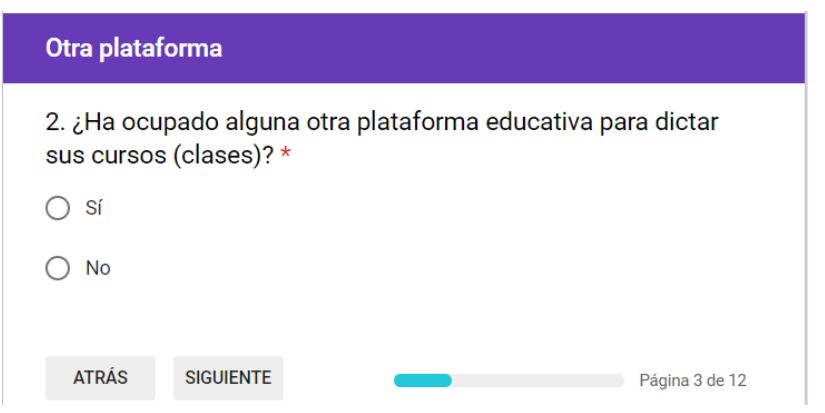

Figura 27 Utilizar otra plataforma

En caso de que la respuesta sea afirmativa se pregunta que otra plataforma utiliza y se procede a finalizar la encuesta con el profesor en cuestión.

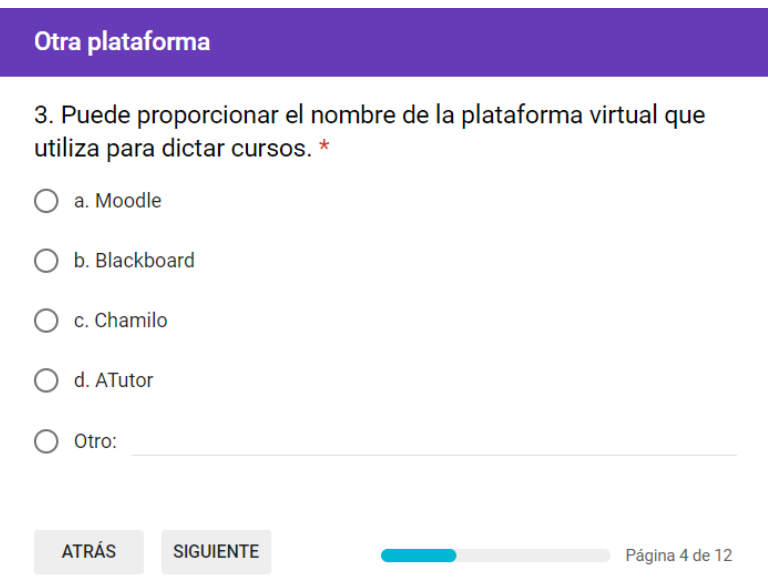

Figura 28 Especificar la otra plataforma

4.2.3 Se pregunta sobre la facilidad de crear cursos o clase en la plataforma Classroom.

2. ¿Es fácil crear cursos en Classroom?

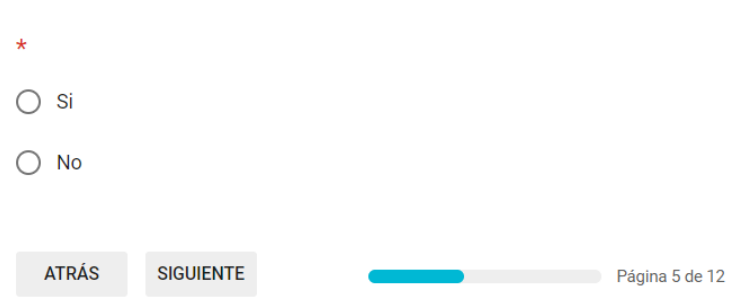

Figura 29 Facilidad para crear cursos

Si la respuesta es negativa se indaga el motivo que genera la dificultad para crear un curso en esta plataforma.

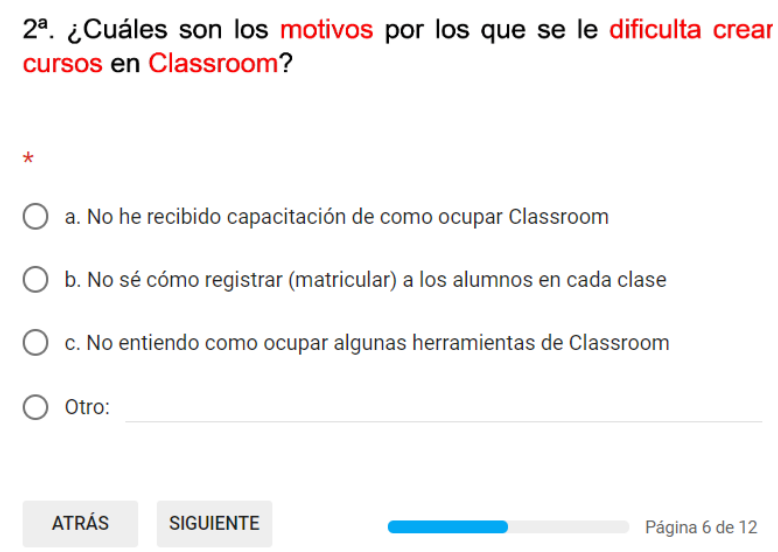

Figura 30 Dificultad para crear cursos

4.2.4 La siguiente pregunta está relacionada con la frecuencia que comparte o habilita actividades y tareas en Classroom el docente.

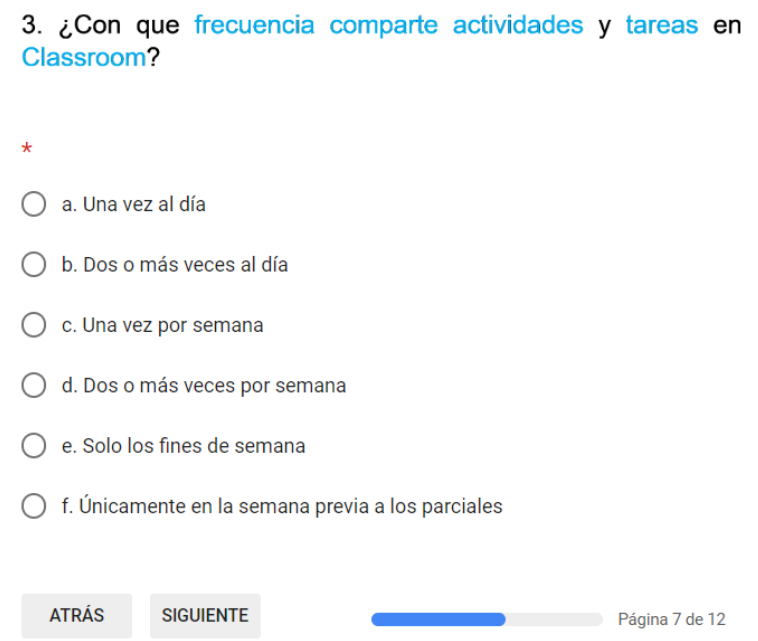

Figura 31 Frecuencia para compartir actividades

4.2.5 Posteriormente se solicita al docente que clasifique de mayor a menor, las dificultades que haya detectado en el empleo de Classroom.

4. Clasifique de mayor a menor importancia las dificultades
encontradas en el uso de Classroom. Siendo 4 la de mayor dificultad y 1 la de menor dificultad.

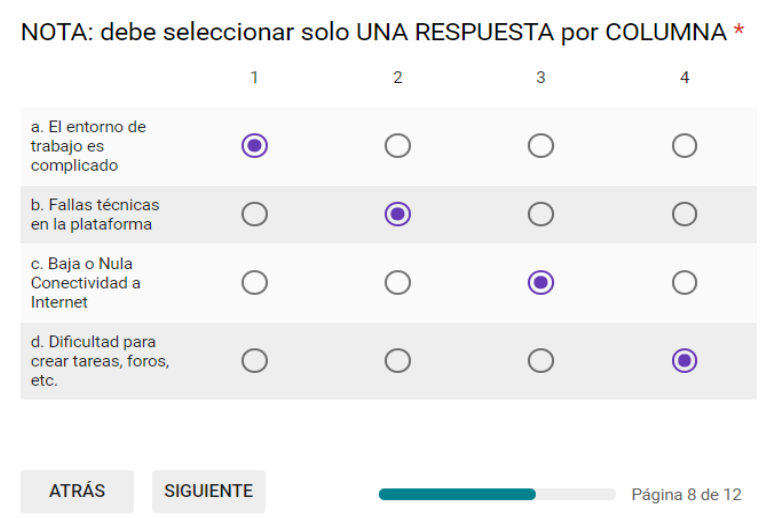

Figura 32 Clasificación de dificultades en Classroom 
4.2.6 En esta pregunta se solicita que clasifique los beneficios que le haya generado utilizar Classroom.

5. De los cuatro beneficios que se mencionan a continuación de la utilización de Classroom, seleccione 1 el que considere más importante y con 4 el que crea menos importante.

NOTA: debe seleccionar solo UNA RESPUESTA por COLUMNA *

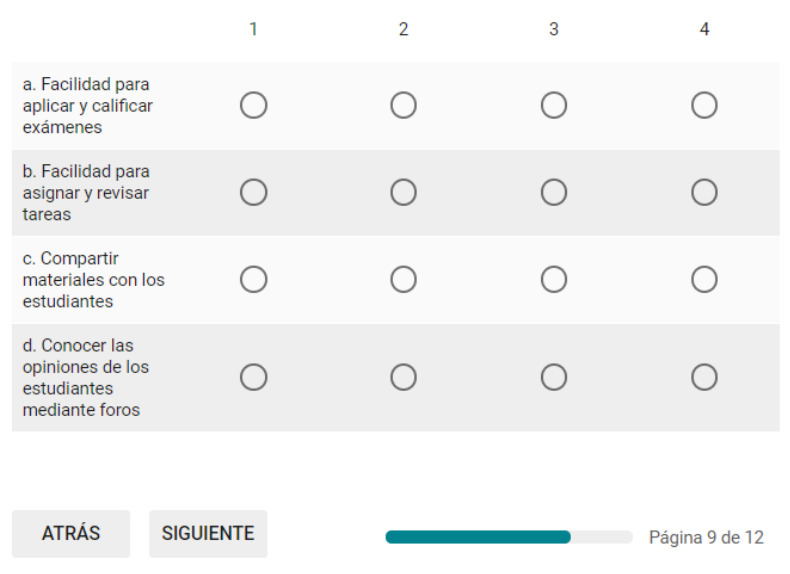

Figura 33 Beneficios de utilizar Classroom

4.2.7 Posteriormente se solicita que especifique que actividades son las emplea en Classroom, con la aclaración de que puede seleccionar dos o más opciones según sea el caso.

6. ¿Cuáles son las actividades que ocupa en Classroom?

(Puede seleccionar dos o más opciones)

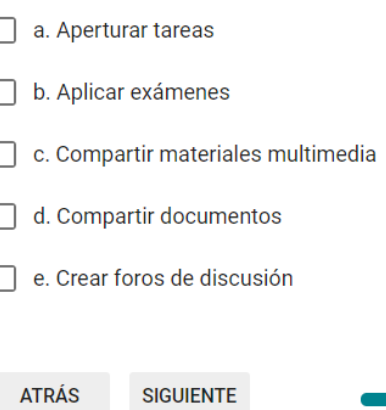

Figura 34 Actividades en Classroom

4.2.8 Se solicita el nivel de satisfacción personal en cuanto al uso de Classroom

\section{7. ¿Qué nivel de satisfacción tiene sobre Classroom?}

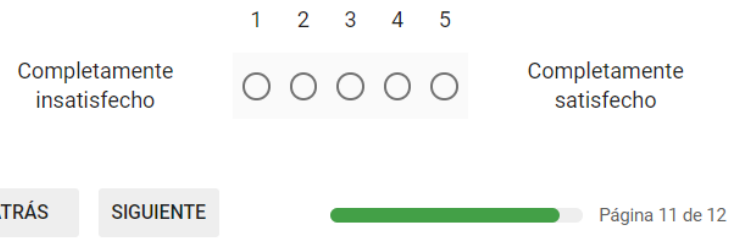

Figura 35 Nivel de satisfacción

4.2.9 Para finalizar se agradece la participación en la encuesta por parte del docente.

\section{Uso de Classroom en la Universidad Tecnológica de Tehuacán (UTTeh)}

\section{¡Gracias por su participación!}

ATRÁS

$$
\text { ENVIAR }
$$

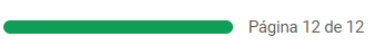

Figura 36 Agradecimiento por participación

\section{Aplicación de la encuesta}

A los estudiantes se aplicó el instrumento de recolección de datos en los laboratorios y aulas de la UTT, con apoyo de los compañeros maestros de los diferentes programas educativos.

A los docentes se les aplicó el instrumento de forma personal y con apoyo de diversos aparatos digitales, propios o de la institución tales como celular o equipo de cómputo, todo en las instalaciones de la UTT.

\section{Resultados}

\section{Classroom en relación con los estudiantes}

De las 250 encuestas aplicadas se obtuvieron los siguientes resultados:

En lo que se refiere a estudiantes que respondieron la encuesta el $55 \%$ pertenece a nivel TSU y el restante $45 \%$ es de nivel ingeniería. En nivel TSU el 25.9\% corresponde a TI área Desarrollo de Software multiplataforma, el $10.6 \%$ a Mecatrónica, el $19.1 \%$ a TI área Infraestructura de Redes Digitales, el $10.8 \%$ a Agricultura Sustentable y Protegida, el $10.8 \%$ a Procesos industriales, el 8.8\% a Desarrollo de Negocios, el $8.8 \%$ a Procesos Bioalimentarios y el $5.2 \%$ a Energías Renovables.

ALFARO-HERRERA, Julio César, VÁZQUEZ-LÓPEZ, José Antonio, GALICIA-GARCÍA, Christian y SÁNCHEZ-DELGADO, Octavio. Utilización de Classroom en la Universidad Tecnológica de Tehuacán (UTTeh). Revista de Tecnologías de la Información y Comunicaciones. 2019 
En lo que se refiere a nivel Ingeniería el $50 \%$ corresponde a Procesos Industriales, el $22.3 \%$ a Agricultura, el $17 \%$ a Procesos Bioalimentarios y el $10.7 \%$ a TI.

Del total de la muestra el $99.6 \%$ a utilizado Classroom, el restante no ha utilizado otra plataforma.

El $99.5 \%$ consideran que utilizar Classroom es fácil e intuitivo. La persona que respondió que no es fácil de utilizar se debe a que no entiende el funcionamiento de todas las diferentes opciones de Classroom

En lo correspondiente a frecuencia de uso de Classroom el porcentaje más alto con un $44.3 \%$ comenta que utiliza Classroom de dos o más veces por semana, por otro lado, el porcentaje más bajo que es el $2 \%$ utiliza Classroom solo los fines de semana.

En cuanto a los motivos y beneficios de utilizar Classroom la mayoría está de acuerdo en la facilidad para entregar tareas, seguido por la rapidez para ingresar a los exámenes, para continuar con la agilidad en la recepción de calificaciones, finalizando con el material disponible para aprender.

El $76.8 \%$ argumenta que no ha participado en ningún foro en Classroom.

En lo que se refiere a facilidad de acceso a Classroom el $84.1 \%$ argumenta que si tienen acceso. Los que no tienen acceso a Classroom en su mayoría $(41 \%)$ comentan que este problema se presenta en las instalaciones de la universidad, seguidos por el $39 \%$ que no tienen servicio de Internet en el hogar.

El principal problema que se les presenta a los estudiantes consiste en que tarda mucho en subir las tareas a Classroom.

El 96.3 coincide que Classroom facilita las actividades escolares.

En lo que se refiere a nivel de satisfacción de la plataforma el 32.9\% completamente satisfecho, el 36.9\% está satisfecho, el $18.3 \%$ esta regularmente satisfecho, el $6.5 \%$ lo suficiente satisfecho y el $5.7 \%$ nada satisfecho.

\section{Classroom en relación con los profesores}

De los 50 profesores encuestas se obtuvieron los siguientes resultados:

El 50\% se encuentra entre los 31-40 años, mientras que el $5 \%$ se encuentra entre los 50 años o más.

Todos los maestros del PE de TI respondieron la encuesta, seguido de los profesores de Procesos Industriales, Desarrollo de Negocios, Mecatrónica y el resto de los PE.

El $65 \%$ de los maestros encuestados imparten la mayor parte de su carga académica a nivel TSU, el resto a nivel Ingeniería.

El $80 \%$ de los encuestados ocupan Classroom. Del 20\% restante a ocupado Moodle o Blackboard como plataforma educativa.

En cuanto a la facilidad de uso, el $90 \%$ argumentan que es sencillo, el 10\% coincide en que se les complica utilizar alguna herramienta.

En lo referente a la frecuencia para compartir actividades y tareas el $43 \%$ indica que lo hace dos o más veces por semana, aunque el $33.3 \%$ indica una vez por semana.

En cuanto a las dificultades con Classroom la mayoría coincide en la baja o nula conectividad a Internet, seguido de que el entorno de trabajo es complicado, además de la dificultad para crear tareas, foros, etc.

En los beneficios el principal es la facilidad para crear y calificar tareas, además de la facilidad para diseñar y calificar exámenes

Las dos actividades principales que hacen los docentes es aperturar tareas y compartir documentos.

En lo que se refiere a nivel de satisfacción el 57 está completamente satisfecho, el $28.6 \%$ se siente bien con Classroom y el $14.4 \%$ considera regular el desempeño de Classroom. 


\section{Conclusiones}

$\mathrm{Al}$ término de la presente investigación se puede concluir que Classroom, como herramienta tecnológica, permite a los estudiantes de la UTT entregar tareas fácilmente, con el inconveniente de que en ocasiones, el tiempo de espera para agregar una tarea o archivo a la plataforma es demasiado, por lo tanto, se permite recomendar a los estudiantes que cuando suban una tarea a la plataforma compriman las imágenes que contiene el archivo para que sea menos pesado, facilite el agregado del mismo y se materialicen los beneficios de ocupar correctamente las TI. Conforme al presente estudio más del $80 \%$ tiene acceso a Internet, por lo tanto este aspecto no es factor para dejar de trabajar y colaborar en línea. Una importante área de oportunidad es que los docentes fomenten el debate mediante plataforma, a través de preguntas detonantes en un foro, mismo que permitirá mejorar la capacidad de análisis, argumentación y síntesis de los estudiantes.

La UTT cuenta con una planta de maestros en la etapa de adultez, motivo por el cual todas los encuestados han utilizado al menos una plataforma educativa para gestionar o elaborar cursos, y es importante destacar que el $80 \%$ ocupa Classroom, esto se debe a que consideran que la plataforma es de fácil uso y cuenta con las elementos necesarios para diversificar el intercambio de información en una clase, por lo tanto, la ocupan en su mayoría dos o más veces por semana. El área de oportunidad detectada la capacitación específica sobre algunas funciones de la plataforma, lo que permitirá la apertura de foros de discusión y compartir materiales de tipo audiovisual.

\section{Agradecimientos}

A la Lic. Yuvanelly López Aguilar por su apoyo en la elaboración del presente artículo.

\section{Referencias}

AYALA, N. J. (2013). TECNOLOGÍAS DE INFORMACIÓN PARA LOS NEGOCIOS EN LA ERA DEL CONOCIMIENTO. México: Digital, del Tecnológico de Monterrey.

CENEVAL. (2000). Estándares de calidad para instrumentos de evaluación educativa. México: CENEVAL.
Coaten, N. (31 de 07 de 2019). Educaweb. Obtenido de Educaweb: https://www.educaweb.com/esp/servicios/mono grafico/formacionvirtual/1181076-a.html

Collazos, A. (29 de 07 de 2019). Revista educación virtual. Obtenido de Revista educación virtual: https://revistaeducacionvirtual.com/archives/94 4

Google. (30 de 07 de 2019). Google Play. Obtenido de Google Play: https://play.google.com/store/apps/details?id=c om.google.android.apps.classroom\&hl=es_MX

Institute, P. M. (2013). Guía de los fundamentos para la dirección de proyectos (guía del PMBOK®), Quinta edición. Estados Unidos: Project Management Institute.

LAUDON Kenneth C., L. J. (2008). Sistemas de información gerencial. México: Pearson.

Matemáticas, D. d. (01 de 08 de 2019). Departamento de Matemáticas de la Universidad de Sonora, México. Obtenido de Departamento de Matemáticas de la Universidad de Sonora, México: http://www.estadistica.mat.uson.mx/Material/el muestreo.pdf

Sampieri, R. (2014). Metodología de la Investigación. Sexta Edición. Mc Graw Hill. México.

http://www.estadistica.mat.uson.mx/Material/el muestreo.pdf

Metodologías y procesos de análisis de software. (22 de 07 de 2019). Obtenido de Metodologías y procesos de análisis de software: http://www.ptolomeo.unam.mx:8080/xmlui/bits tream/handle/132.248.52.100/175/A5\%20Cap\% C3\%ADtulo\%202.pdf? sequence $=5$ 